\title{
Prognostic evaluation of preoperative serum C-reactive protein concentration in patients with epithelial ovarian cancer
}

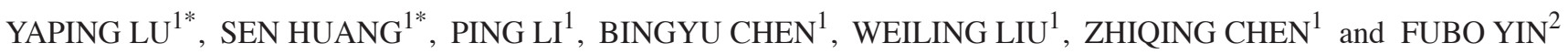 \\ ${ }^{1}$ Department of Obstetrics and Gynecology, Haimen City People's Hospital, Haimen, Jiangsu 226100; \\ ${ }^{2}$ Department of Obstetrics and Gynecology, Provincial Hospital Affiliated to Shandong University, \\ Jinan, Shandong 250021, P.R. China
}

Received June 11, 2014; Accepted February 16, 2015

DOI: $10.3892 / \mathrm{etm} .2015 .2350$

\begin{abstract}
The aim of the present study was to evaluate correlations between preoperative C-reactive protein (CRP) serum concentration and the clinical-pathological parameters of epithelial ovarian cancer (EOC), as well as cancer antigen 125 (CA125) concentration, in patients with EOC. The preoperative serum CRP concentrations of 107 patients with EOC were compared with values of 44 control patients and defined as positive if values were $>8 \mathrm{mg} / \mathrm{l}$. Correlations between CRP expression and serum CA125 concentrations, International Federation of Gynecologists and Obstetricians (FIGO) staging, lymph node metastasis, ascites and size of postoperative residual lesions were evaluated for the EOC cases. A total of $69 \%$ (74/107) of the EOC cases were CRP-positive, with a mean serum CRP concentration of $14.32 \mathrm{mg} / 1$ (versus $2.18 \mathrm{mg} / \mathrm{l}$ in the control patients $)(\mathrm{P}<0.001)$. A rank correlation analysis showed a positive correlation between serum CRP and CA125 concentrations $(\mathrm{P}<0.001)$. The five-year survival rate of the CRP-positive patients with EOC was significant lower than that of the patients that were CRP-negative (33.3 vs. $75.8 \%, \mathrm{P}<0.001)$. Non-parametric and multivariate analyses showed significant correlations between CRP concentrations and FIGO staging $(\mathrm{P}=0.001)$, lymph node metastasis $(\mathrm{P}=0.001)$ and ascites $(\mathrm{P}=0.033)$. In contrast to previous reports, the mean CRP serum concentration of the CRP-positive patients with EOC also correlated significantly with the CA125 values, and the increases in the CRP serum concentration in the Chinese patients were lower than those described for Caucasian EOC cases. In conclusion, the CRP serum concentration may be a
\end{abstract}

Correspondence to: Mrs. Zhiqing Chen, Department of Obstetrics and Gynecology, Haimen City People's Hospital, 253 Renmin West Road, Haimen, Jiangsu 226100, P.R. China

E-mail: 1wyanhao2008@sina.cn

*Contributed equally

Key words: epithelial ovarian cancer, C-reactive protein, cancer antigen 125, clinicopathological data, prognosis useful clinical marker, solely or in combination with CA125, in patients with EOC.

\section{Introduction}

The incidence of ovarian cancer is gradually rising and its mortality rate ranks first among the gynecological malignant tumors; the majority of cases are epithelial ovarian cancer (EOC) derived from the ovarian epithelial tissue (1). The pathogenesis and development of ovarian cancer have been closely linked to inflammatory processes associated with several cytokines $(2,3)$, such as vascular endothelial growth factor (4) interleukin-6 (5) and interleukin-12 (6). Among the already established prognostic indicators, ovarian tumor stage and postoperative residual tumor mass at primary cytoreductive surgery have been shown to most reliably predict the outcome of patients with ovarian cancer (7). Clinical decisions regarding adjuvant therapies are largely based on the International Federation of Gynecologists and Obstetricians (FIGO) stage and tumor grade, particularly in the early stages of the disease (8). With the progress in the surgical techniques, management of the perioperative period and range of combined treatments, the survival rate of patients with ovarian cancer has been increased significantly; however, the high recurrence rate following treatment remains the primary reason for poor prognoses (9). Reliable indicators for the identification of those patients that are at high risk of recurrence or mortality in the early stages of the disease would be beneficial for the evaluation of the prognosis of patients with ovarian cancer and for the development of specific measures to counteract unfavorable outcomes.

C-reactive protein (CRP) is an acute-phase protein expressed as an acute response to infections and tissue injuries, and increases in its expression are often associated with multiple system and organ diseases (10). At present, the theory of a causal association between inflammation and the innate immune system regarding cancer is widely accepted. Numerous malignant tumors occur at sites of infection, chronic irritation and inflammation, and 15-20\% of human tumors are associated with inflammations (11). Several studies have shown that patients with cancer have higher circulating CRP levels than healthy controls prior to clinical diagnosis (3-5 years) (12-15). In addition, the increase in serum CRP has 
been reported to be associated with poor prognosis in esophageal, liver, colon, lung and cervical cancer (16-20). Among the various gynecological tumor markers, such as $\alpha$-fetoprotein and human epididymis protein-4 (21), cancer antigen 125 (CA125) is the most specific and sensitive marker for EOC detection (22), particularly for recurrent tumors $(21,22)$. It has been reported that there are considerable ethnic differences regarding the five-year survival rate of patients with EOC; in Asian females, the five-year survival rate was found to be almost double that of Caucasians in a survey performed in the USA between 1992 and 1997 (25). There are few reports, however, investigating whether the serum CRP concentration of patients with ovarian cancer in China complies with that in European patients and those from the USA; therefore, the aim of the present study was to investigate a correlation between the serum CRP concentrations and clinicopathological parameters of Chinese patients with EOC, using CA125 as a positive control marker.

\section{Materials and methods}

Characteristics of patients. Between January 2006 and March 2010, 107 patients with EOC underwent surgery at the Departments of Obstetrics and Gynecology of the Provincial Hospital Affiliated to Shandong University (Jinan, China; $\mathbf{n = 7 1 )}$ and the Haimen City People's Hospital (Haimen, China; n=36). Diagnoses were confirmed by the pathology results. All the patients were classified by FIGO (2000) standard staging (26), and the age of the patients at the first visit was 34-79 years (mean, 55.28 \pm 10.34 years). The control group comprised females with benign ovarian tumors $[n=32$; age, 32-72 years (mean age, 51.38 \pm 9.05 years)], including simple ovarian cyst, benign serous or mucinous cystadenoma, and healthy adult females $[n=12$; age, $38-61$ years $(49.50 \pm 6.53$ years) $]$. There was no significant age difference between the groups.

Enrollment criteria. For enrollment in the study, patients had to meet the following criteria: i) Ovarian cancer without surgical contraindication and EOC confirmed by postoperative pathology; and ii) normal white blood cell count and neutrophil ratios, as shown by routine blood examination on admission. Patients were excluded due to infection, trauma, coronary heart disease, hypertension and connective tissue diseases, as these conditions could result in elevated CRP concentrations. The study protocol was approved by the Ethics Committee of Haimen City People's Hospital and the Provincial Hospital Affiliated to Shandong University, and all participants provided their written informed consent to participate in the study.

Measurement methods. On the second day after admission, $6 \mathrm{ml}$ morning fasting blood was collected from each patient and the serum of $3 \mathrm{ml}$ was used for CRP quantification via nephelometry rates (High-Sensitivity CRP kit; Beckman Coulter, Miami, FL, USA). With the development of a high-sensitivity assay method, the associations between inflammation and disease risk allow a wide spectrum of CRP levels, particularly in the range of concentrations previously widely considered 'normal', i.e. levels $<10 \mathrm{mg} / \mathrm{l}$. Due to the intra-assay variability (1.64-3.34\%), CRP serum levels $\leq 8 \mathrm{mg} / \mathrm{l}$ were defined as the normal range in the two hospitals in the present study. The serum of another $3 \mathrm{ml}$ blood was used to determine the content of CA125 via electrochemiluminescence (Roche Diagnostics $\mathrm{GmbH}$, Manneheim, Germany), and the CA125 normal reference value was set to $0-35 \mathrm{U} / \mathrm{ml}$.

Surgery and postoperative adjunctive therapy. A total of 69 patients received surgery without residual tumor or residual lesions $<2 \mathrm{~cm} ; 38$ patients received subtotal tumor resections with residual lesions $\geq 2 \mathrm{~cm}$. Sixty-five cases underwent pelvic and para-aortic lymph node dissection: 21 patients had lymph node metastasis and 44 cases were without metastasis. Intraperitoneal combined with intravenous chemotherapy or simple intravenous chemotherapy was started 7 days after surgery once every 4 weeks, and regular follow-ups were performed for 11-74 months with a mean duration of 28.5 months.

Statistical analysis. SPSS 17.0 statistical software was used for the statistical analyses, and the measurement data are presented as the mean \pm standard deviation or median. Comparisons of measurement data were performed using the Student's t-test, rank sum test or analysis of variance, and count data were compared using the $\chi^{2}$ test. Single or multiple factors were compared using logistic regression analyses. The correlation of CRP with CA125 was analyzed by a Spearman rank correlation analysis. $\mathrm{P}<0.05$ was considered to indicate a statistically significant difference.

\section{Results}

Patients' characteristics. According to the FIGO staging criteria, 29 cases were in stage I (27.10\%), 14 cases in stage II (13.08\%), 44 cases in stage III (41.12\%) and 20 cases in stage IV (18.69\%). The analysis of pathological type revealed that 43 cases were serous cystadenocarcinoma (40.19\%); 38 cases were mucinous cystadenocarcinoma $(35.51 \%)$ and 35 cases were other types of epithelial cancers (32.71\%), including endometrial cancer, clear cell carcinoma, transitional cell carcinoma and epithelial cancer that could not be classified due to tissue necrosis. Ascites occurred in 42 cases (39.25\%), and 65 cases (60.75\%) were without ascites. For histological cell grading, 28 cases were in the G1 phase (26.17\%), 44 cases in the G2 phase (41.12\%) and 35 cases in the $\mathrm{G} 3$ phase $(32.71 \%)$.

Preoperative serum CRP and CA125 concentrations of patients with EOC. In the present study, $62 \%$ of the patients $(66 / 107)$ were positive for $\mathrm{CRP}(\mathrm{CRP}>8 \mathrm{mg} / \mathrm{l})$ in the EOC group, while no positive results were found in the control group. The mean preoperative serum CRP concentration of the patients with EOC was $14.32 \mathrm{mg} / \mathrm{l}(1.02-163.00 \mathrm{mg} / \mathrm{l})$, which was $\sim 6.6$-fold that of the ovarian benign tumor group [2.175 mg/l (1.00-4.59 mg/l)] $(\mathrm{P}<0.001)$, as well as $\sim 9.7$-fold the serum concentration of the healthy adult females $[1.47 \mathrm{mg} / \mathrm{l}(1.00-3.16 \mathrm{mg} / \mathrm{l})](\mathrm{P}<0.001)$. The serum concentration of CA125 in the EOC group was $475.90 \mathrm{U} / 1$ (16.26-5,000 U/1), whereas in the benign ovarian tumor group it was $23.85 \mathrm{U} / 1$ (7.74-61.31 U/1) and in the healthy adult females it was $8.77 \mathrm{U} / 1(1.35-18.33 \mathrm{U} / \mathrm{l})(\mathrm{P}<0.05)$. The CA125 serum concentration in the EOC group was 20 -fold that of the benign ovarian tumor group and 54-fold that of the 
Table I. Correlation of serum CRP concentration with the clinicopathological parameters of patients with epithelial ovarian cancer.

\begin{tabular}{|c|c|c|c|c|c|}
\hline $\begin{array}{l}\text { Clinicopathological } \\
\text { parameter }\end{array}$ & $\mathrm{n}$ & CRP level, mg/l & $\begin{array}{l}\text { P-value }{ }^{\mathrm{a}} \\
\text { ANOVA }\end{array}$ & $\begin{array}{c}\text { CRP-positive rate }{ }^{\mathrm{b}} \\
\mathrm{n}(\%)\end{array}$ & $\begin{array}{l}\text { P-value }{ }^{a}, \\
\text { ANOVA }\end{array}$ \\
\hline \multicolumn{6}{|l|}{ Age, years } \\
\hline$>50$ & 63 & $22.24 \pm 36.39$ & \multirow[t]{2}{*}{0.283} & $35(56)$ & \multirow[t]{2}{*}{0.081} \\
\hline$\leq 50$ & 44 & $29.27 \pm 23.93$ & & $31(54)$ & \\
\hline \multicolumn{6}{|l|}{ FIGO stage } \\
\hline $\mathrm{I}$ & 29 & $4.61 \pm 3.06$ & \multirow[t]{4}{*}{$<0.001$} & $4(14)$ & \multirow[t]{4}{*}{$<0.001$} \\
\hline II & 14 & $10.81 \pm 4.48$ & & $10(71)$ & \\
\hline III & 44 & $35.48 \pm 36.98$ & & $35(91)$ & \\
\hline IV & 20 & $51.04 \pm 33.23$ & & $17(80)$ & \\
\hline \multicolumn{6}{|l|}{ Histological grading } \\
\hline G1 & 28 & $12.03 \pm 14.21$ & \multirow[t]{3}{*}{0.005} & $11(39)$ & \multirow[t]{3}{*}{0.165} \\
\hline G2 & 44 & $26.75 \pm 29.70$ & & $30(68)$ & \\
\hline G3 & 35 & $38.66 \pm 41.58$ & & $25(71)$ & \\
\hline \multicolumn{6}{|l|}{ Type } \\
\hline Pathological serous cystadenocarcinoma & 43 & $29.93 \pm 35.93$ & \multirow[t]{3}{*}{0.089} & $28(65)$ & \multirow[t]{3}{*}{0.118} \\
\hline Mucinous cystadenocarcinoma & 38 & $13.24 \pm 10.93$ & & $22(58)$ & \\
\hline Other types of epithelial carcinoma & 26 & $32.19 \pm 34.18$ & & $16(62)$ & \\
\hline \multicolumn{6}{|l|}{ Ascites } \\
\hline With & 42 & $36.01 \pm 34.92$ & \multirow[t]{2}{*}{0.014} & $36(86)$ & \multirow[t]{2}{*}{0.026} \\
\hline Without & 65 & $20.36 \pm 29.63$ & & $30(46)$ & \\
\hline \multicolumn{6}{|l|}{ Tumor resection } \\
\hline Residual tumor diameter $<2 \mathrm{~cm}$ & 69 & $21.14 \pm 27.43$ & \multirow[t]{2}{*}{0.006} & $39(57)$ & \multirow[t]{2}{*}{0.004} \\
\hline Residual tumor diameter $\geq 2 \mathrm{~cm}$ & 38 & $40.04 \pm 39.98$ & & $27(71)$ & \\
\hline \multicolumn{6}{|l|}{ Lymph node metastasis } \\
\hline Positive & 21 & $30.79 \pm 36.99$ & \multirow[t]{2}{*}{0.002} & $17(81)$ & \multirow[t]{2}{*}{0.004} \\
\hline Negative & 44 & $10.14 \pm 15.31$ & & $15(34)$ & \\
\hline
\end{tabular}

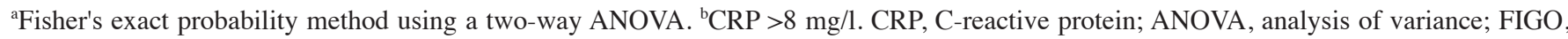
International Federation of Gynecologists and Obstetricians.

healthy adult females. A rank correlation analysis of preoperative serum CRP and CA125 concentrations in the EOC group revealed that the Spearman correlation coefficient (rs) was 0.603 ( $\mathrm{P}<0.001$, two-way), suggesting that CRP was positively correlated with CA125; however, no significant correlation between the CRP and CA125 concentrations existed in the control group (rs=-0.095, $\mathrm{P}=0.708)$.

Correlation of EOC serum CRP concentrations with clinical and pathological parameters. It was next analyzed whether elevated preoperative serum CRP concentrations correlated with the clinicopathological parameters of the patients with EOC. As shown in Table I, the serum concentrations of CRP correlated with histological grading, FIGO staging, ascites, tumor size and lymph node metastasis, whereas only positive serum CRP did not correlate with histological grading. No significant difference was found in the serum CRP concentrations between different ages and different pathological tumor types.

Logistic regression analyses of single and multiple factors affecting CRP serum concentrations. Based on the results of single factor analyses, a positive serum CRP value was correlated with FIGO tumor staging, histological grading, presence of ascites and lymph node metastasis (Table II), while CA125 had no effect on CRP-positive rates. When the factors that correlated with CRP in the single factor analyses as independent variables were used in a multiple factor logistic regression analysis it was found that the FIGO tumor staging, presence of ascites and lymph node metastasis were correlated with CRP-positive rates (Table III).

Correlation of CRP expression with the five-year survival rate of patients with EOC. The overall five-year survival rate of patients with serum CRP $\leq 8 \mathrm{mg} / \mathrm{l}$ was $75.8 \%$, compared with a survival rate of $33.3 \%$ for patients with CRP concentrations $>8 \mathrm{mg} / \mathrm{l}(\mathrm{P}<0.001)$ (Fig. 1).

\section{Discussion}

McSorley et al (27) found that the serum concentration of CRP in females with ovarian cancer was enhanced, indicating chronic inflammation during the development of 
Table II. Single factor logistic regression analysis for C-reactive protein expression.

$95 \% \mathrm{CI}$ of OR

\begin{tabular}{lcccccccr}
\cline { 6 - 9 } Factor & $\mathrm{B}$ & $\mathrm{SE}$ & Wald & df & P-value & OR & Lower & Upper \\
\hline Histological grading & -1.151 & 0.794 & 3.147 & 1 & 0.047 & 0.163 & 0.537 & 2.517 \\
Pathological type & 0.360 & 0.366 & 0.964 & 1 & 0.326 & 1.433 & 0.699 & 2.938 \\
Ascites & -1.732 & 0.805 & 4.633 & 1 & 0.031 & 0.177 & 0.037 & 0.857 \\
Residual tumor & -0.296 & 0.661 & 0.201 & 1 & 0.654 & 0.744 & 0.204 & 2.716 \\
CA125 & -0.633 & 0.958 & 0.436 & 1 & 0.509 & 0.531 & 0.081 & 3.473 \\
FIGO stage & -1.287 & 0.412 & 9.771 & 1 & 0.002 & 0.276 & 0.123 & 0.619 \\
Lymph node & 2.144 & 0.663 & 10.468 & 1 & 0.001 & 8.537 & 2.329 & 31.298 \\
Age & 0.383 & 0.583 & 0.431 & 1 & 0.511 & 1.467 & 0.468 & 4.599
\end{tabular}

CI, confidence interval; OR, odds ratio; SE, standard error; df, degrees of freedom; CA125, cancer antigen 125; FIGO, International Federation of Gynecologists and Obstetricians.

Table III. Multiple factor logistic regression analysis for C-reactive protein expression.

$95 \%$ CI of OR

\begin{tabular}{lccccccrr}
\cline { 5 - 8 } Factor & $\mathrm{B}$ & $\mathrm{SE}$ & Wald & $\mathrm{df}$ & P-value & OR & Lower & Upper \\
\hline FIGO stage & 1.381 & 0.412 & 11.227 & 1 & 0.001 & 3.980 & 1.774 & 8.928 \\
Ascites & 1.767 & 0.826 & 4.571 & 1 & 0.033 & 5.852 & 1.158 & 29.559 \\
Lymph node metastasis & -2.087 & 0.646 & 10.420 & 1 & 0.001 & 0.124 & 0.035 & 0.441 \\
\hline
\end{tabular}

CI, confidence interval; OR, odds ratio; SE, standard error; df, degrees of freedom; FIGO, International Federation of Gynecologists and Obstetricians.

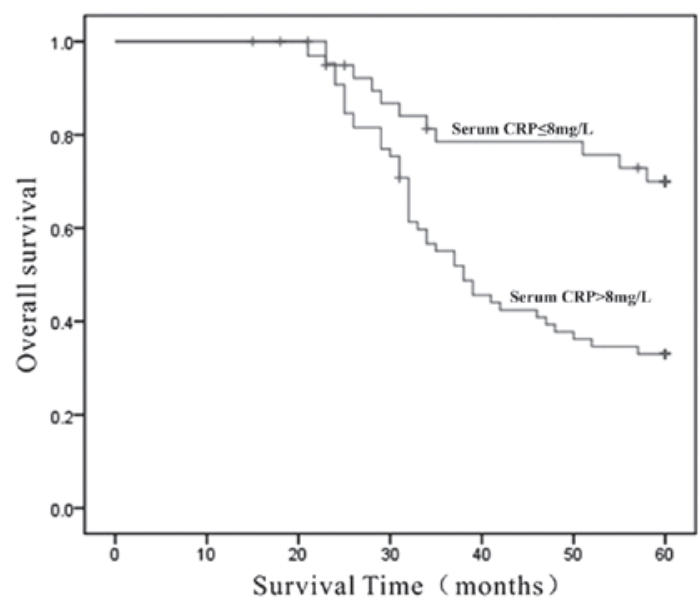

Figure 1. Kaplan-Meier curves for overall survival of patients with ovarian cancer broken down by serum CRP. CRP, C-reactive protein concentration.

ovarian cancer. Other gynecological inflammatory diseases, such as pelvic inflammation, endometriosis and polycystic ovarian syndrome, may progress into ovarian cancer, which indirectly supports the interaction of inflammation and cancer (28). Ovulation is basically an inflammatory process, which includes repair cycles of the ovarian cortex wound with concomitant healing. It has been suggested that persistent ovulation is a potential inducer of ovarian cancer; this is supported by the fact that anovulatory factors, including oral contraceptives, pregnancy and lactation, can greatly reduce the risk of ovarian cancer (29). Cancer induces nonspecific inflammation, leading to the release of a variety of pro-inflammatory mediators and factors (30). These nonspecific inflammatory reactions produced by tumor tissue necrosis and/or local tissue injury can induce the liver cells to synthesize CRP, which is then released into the serum. Several reports have confirmed that serum CRP levels are elevated in patients with malignant tumors, and the elevation is associated with the malignancy degree, increase in tumor metastasis rate and decrease in postoperative survival rate $(16-20,31)$. In the present study it was demonstrated that preoperative serum CRP concentrations correlated with tumor FIGO staging, lymph node metastasis and ascites formation among the 107 patients with EOC, which is in line with the previous reports (32-34). Furthermore, it was found in this study that the average CRP concentration in Chinese patients with EOC was $14.32 \mathrm{mg} / \mathrm{l}$, which was lower than the value reported for Caucasian cases (36 mg/l) (32), which may reflect ethnical variations; however, the same study (32) also reported that enhanced serum CRP concentrations correlated with an unfavorable prognosis, which has been confirmed by the present finding that CRP-positive patients with EOC had significantly lower five-year survival rates. In the 107 cases 
of EOC in the present study, the mean preoperative serum CA125 concentration was $475.90 \mathrm{U} / \mathrm{ml}$, and the value in the EOC group was significantly higher than that in the benign ovarian tumor group $(23.85 \mathrm{U} / \mathrm{ml})(\mathrm{P}<0.001)$. Based on a rank correlation analysis of serum CRP and CA125 concentrations, a positive correlation was found $(\mathrm{P}<0.001)$, which is also in contrast to previous reports $(32,33)$.

In conclusion, the CRP serum concentrations of patients with EOC were significantly enhanced and correlated with FIGO staging, lymph metastases and the occurrence of ascites, as well as with CA125 serum concentrations. Preoperative CRP serum concentration analyses could be a useful parameter for evaluating the severity of EOC and treatment adjustments, and could be utilized to monitor the treatment success in combination with the measurement of serum CA125 concentration.

\section{Acknowledgements}

The authors would like to thank Mr. Xiancai Ni, Mr. Qiongcheng Yan and Ms. Jumei Gu from the Haimen City People's Hospital for coordinating the study and for their assistance with data collection.

\section{References}

1. Wong KH, Mang OW, Au KH and Law SC: . Incidence, mortality, and survival trends of ovarian cancer in Hong Kong, 1997 to 2006: a population-based study. Hong Kong Med J 18: 466-474, 2012.

2. Helzlsouer KJ, Erlinger TP and Platz EA: C-reactive protein levels and subsequent cancer outcomes: results from a prospective cohort study. Eur J Cancer 42: 704-707, 2006.

3. Balkwill $\mathrm{F}$ and Mantovani A: Inflammation and cancer: back to Virchow? Lancet 357: 539-545, 2001.

4. Hefler LA, Zeillinger R, Grimm C, et al: Preoperative serum vascular endothelial growth factor as a prognostic parameter in ovarian cancer. Gynecol Oncol 103: 512-517, 2006.

5. Tempfer C, Zeisler H, Sliutz G, et al: Serum evaluation of interleukin 6 in ovarian cancer patients. Gynecol Oncol 66: 27-30, 1997

6. Zeimet AG, Widschwendter M, Knabbe C, et al: Ascitic interleukin-12 is an independent prognostic factor in ovarian cancer. J Clin Oncol 16: 1861-1868, 1998.

7. Friedlander ML: Prognostic factors in ovarian cancer. Semin Oncol 25: 305-314, 1998

8. Winter-Roach BA, Kitchener HC and Lawrie TA: Adjuvant (post-surgery) chemotherapy for early stage epithelial ovarian cancer. Cochrane Database Syst Rev 3: CD004706, 2012.

9. Marchetti C1, Pisano C, Facchini G, et al: First-line treatment of advanced ovarian cancer: current research and perspectives. Expert Rev Anticancer Ther 10: 47-60, 2010.

10. Marnell L, Mold C and Du Clos TW: C-reactive protein: ligands, receptors and role in inflammation. Clin Immunol 117: 104-111, 2005.

11. Allavena P, Garlanda C, Borrello MG, Sica A and Mantovani A: Pathways connecting inflammation and cancer. Curr Opin Genet Dev 18: 3-10, 2008.

12. Lundin E, Dossus L, Clendenen T, et al: C-reactive protein and ovarian cancer: a prospective study nested in three cohorts (Sweden, USA, Italy). Cancer Causes Control 20: 1151-1159, 2009.

13. Siemes C, Visser LE, Coebergh JW, et al: C-reactive protein levels, variation in the C-reactive protein gene, and cancer risk: the Rotterdam Study. J Clin Oncol 24: 5216-5222, 2006.
14. Il'yasova D, Colbert LH, Harris TB, et al: Circulating levels of inflammatory markers and cancer risk in the health aging and body composition cohort. Cancer Epidemiol Biomarkers Prev 14: 2413-2418, 2005

15. Trichopoulos D, Psaltopoulou T, Orfanos P, Trichopoulou A and Boffetta P: Plasma C-reactive protein and risk of cancer: a prospective study from Greece. Cancer Epidemiol Biomarkers Prev 15: 381-384, 2006.

16. Gockel I, Dirksen K, Messow CM and Junginger T: Significance of preoperative C-reactive protein as a parameter of the perioperative course and long-term prognosis in squamous cell carcinoma and adenocarcinoma of the oesophagus. World J Gastroenterol 12: 3746-3750, 2006.

17. Hashimoto K, Ikeda Y, Korenaga D, et al: The impact of preoperative serum C-reactive protein on the prognosis of patients with hepatocellular carcinoma. Cancer 103: 1856-1864, 2005.

18. McMillan DC, Canna K and McArdle CS: Systemic inflammatory response predicts survival following curative resection of colorectal cancer. Br J Surg 90: 215-219, 2003.

19. Brown DJ, Milroy R, Preston T and McMillan DC: The relationship between an inflammation-based prognostic score (Glasgow Prognostic Score) and changes in serum biochemical variables in patients with advanced lung and gastrointestinal cancer. J Clin Pathol 60: 705-708, 2007.

20. Polterauer S, Grimm C, Tempfer C, et al: C-reactive protein is a prognostic parameter in patients with cervical cancer. Gynecol Oncol 107: 114-117, 2007.

21. Anastasi E, Marchei GG, Viggiani V, et al: HE4: a new potential early biomarker for the recurrence of ovarian cancer. Tumour Biol 31: 113-119, 2010.

22. Klug TL, Bast RC Jr, Niloff JM Knapp RC and Zurawski VR Jr : Monoclonal antibody immunoradiometric assay for an antigenic determinant (CA 125) associated with human epithelial ovarian carcinomas. Cancer Res 44: 1048-1053, 1984.

23. Schilthuis MS, Aalders JG, Bouma J, et al: Serum CA 125 levels in epithelial ovarian cancer: relation with findings at second-look operations and their role in the detection of tumour recurrence. Br J Obstet Gynaecol 94: 202-207, 1987.

24. Santillan A, Garg R, Zahurak ML, et al: Risk of epithelial ovarian cancer recurrence in patients with rising serum CA-125 levels within the normal range. J Clin Oncol 23: 9338-9343, 2005.

25. Howe HL, Tung KH, Coughlin S, Jean-Baptiste R and Hotes J: Race/ethnic variations in ovarian cancer mortality in the United States, 1992-1997. Cancer 97 (10 Suppl): 2686-2693, 2003.

26. Benedet JL, Bender H, Jones H III, Ngan HY and Pecorelli S: FIGO staging classifications and clinical practice guidelines in the management of gynecologic cancers. FIGO Committee on Gynecologic Oncology. Int J Gynaecol Obstet 70: 209-262, 2000.

27. McSorley MA, Alberg AJ, Allen DS, et al: C-reactive protein concentrations and subsequent ovarian cancer risk. Obstet Gynecol 109: 933-941, 2007.

28. Ness RB: Endometriosis and ovarian cancer: thoughts on shared pathophysiology. Am J Obstet Gynecol 189: 280-294, 2003.

29. Tung KH, Wilkens LR, Wu AH, et al: Effect of anovulation factors on pre- and postmenopausal ovarian cancer risk: revisiting the incessant ovulation hypothesis. Am J Epidemiol 161: 321-329, 2005.

30. Shacter E and Weitzman SA: Chronic inflammation and cancer. Oncology (Williston Park) 16: 217-226, 2002.

31. McMillan DC, Elahi MM, Sattar N, et al: Measurement of the systemic inflammatory response predicts cancer-specific and non-cancer survival in patients with cancer. Nutr Cancer 41: 64-69, 2001.

32. Hefler LA, Concin N, Hofstetter G, et al: Serum C-reactive protein as independent prognostic variable in patients with ovarian cancer. Clin Cancer Res 14: 710-714, 2008.

33. Hefler-Frischmuth K, Hefler LA, Heinze G, et al: Serum C-reactive protein in the differential diagnosis of ovarian masses. Eur J Obstet Gynecol Reprod Biol 147: 65-68, 2009.

34. Lu Y, Yin F and Chen Z: Prognostic value of preoperative serum C-reactive protein in patients with epithelial ovarian cancer. Progress in Obstetrics and Gynecology 17: 489-492, 2008. 